\title{
TEST RESULTS FROM THE SMES PROOF OF PRINCIPLE EXPERIMENT
}

J. M. Pfotenhauer, M. K. Abdelsalam, F. Bodker, D. Hutleston, Z. Jiang,

O. D. Lokken, D. Scherbarth*, B. Tao, and D. Yu

Applied Superconductivity Center

University of Wisconsin

1500 Johnson Drive

Madison, WI 53706

\author{
*Westinghouse Science \& Technology Center \\ Building 501-3E9 \\ 1310 Beulah Road \\ Pittsburgh, PA 15235
}

$\underline{\text { Abstract }}$

A Proof of Principle Experiment (POPE) has been constructed and conducted to demonstrate the stability and operation of the SMES conductor in the SMES-WISC team's Engineering Test Model (ETM) design. The experimental facility includes: a $100 \mathrm{kA} \mathrm{DC}$ power supply; a 4 tesla, 1 meter bore, background field split solenoid; a three turn-1 meter diameter test coil of the ETM conductor, a dewar for operation of the solenoid and test coil at $1.8 \mathrm{~K}$ and 1 atmosphere, and support systems for vacuum, helium supply and recovery, and data acquisition. The test facility exactly duplicates the electric, magnetic and thermal conditions expected for the ETM conductor. We report on measurements of conductor stability vs. transport current, applied magnetic field, and cooling from liquid helium. The measurements characterize the conductor's stability against finite length traveling normal zones, and against quenches resulting from transient normal zones. The data qualify the conductor for dependable use at $50 \mathrm{kA}, 4$ tesla and $1.8 \mathrm{~K}$.

\section{Introduction}

Conductor stability plays a vital role in the design of any superconducting magnet. However, in contrast to the ease of fabrication and repairability typical of laboratory scale magnets, or the stringent space requirements typical of accelerator magnets, the key factor driving the conductor design for the large SMES magnet is absolute dependability over a 50 year lifetime of constant use. This report details the Proof of Principle Experiment (POPE) which demonstrates that the conductor design chosen by the SMES/WISC team for the ETM will provide this dependability.

The POPE is fundamentally a stability test for the ETM conductor, and as such is designed and built to duplicate the exact conditions which the conductor will see in the ETM. These conditions include continuous DC currents of up to $50 \mathrm{kA}$, magnetic fields of up to 4 tesla, direct cooling by an annular bath of liquid helium at $1.8 \mathrm{~K}$ and 1 atmosphere, and high strength aluminum structural support. The primary goals of the POPE are to verify the stability calculations used to design

Manuscript received September 24, 1990. the conductor and to thereby qualify it for use at $50 \mathrm{kA}, 4$ tesla and $1.8 \mathrm{~K}$. The conductor is comprised of a $2.54 \mathrm{~cm}$ diameter high purity aluminum stabilizer with eight $\mathrm{NbTi}-\mathrm{Cu}$ strands of $2.8 \mathrm{~mm}$ diameter soldered into helical grooves along the surface of the aluminum stabilizer.

In the POPE the conductor is tested for stability at $50 \mathrm{kA}$ against a runaway normal zone expansion, or quench, caused by local, transient, thermal disturbances. This is commonly referred to as transient stability. In addition, the POPE provides a test for stability against a finite length traveling normal zone. This type of instability is specific to composite superconductors with long transverse current diffusion times. In the SMES/WISCETM conductor, for example, the current diffusion time constant is $\sim 0.3$ seconds. In the current sharing mode the initial axial current flow is through a small cross-sectional area. The result is that the $I^{2} R$ heat generation at the front end of a normal zone is larger than that behind the front. In addition, the good thermal conductivity of the high purity aluminum stabilizer allows for rapid heat flow ahead of the normal zone. This in turn drives the temperature of the superconducting strands above their current sharing temperature and the normal zone becomes self perpetuating. In summary, the factors of axial thermal conduction and time-dependent cooling to the helium combine with the current diffusion process to generate a normal zone that propagates rapidly forward but which recovers at the back end; thus a traveling normal zone. Theoretical and numerical descriptions of a finite length traveling normal zone and the so named "dynamic stability" associated with this type of propagation are available ${ }^{1-4}$ and are primary considerations in the design of the SMES/WISC-ETM conductor. As is shown below, the Proof of Principle Experiment directly verifies the dynamic stability calculations for the ETM conductor, and demonstrates the existence of a traveling normal zone.

\section{Equipment}

At the heart of the POPE is a three-turn winding, or test coil, of the $50 \mathrm{kA}$ ETM conductor. The test coil, supported by a high strength aluminum structure identical in material and crosssection to that for the ETM, is wound one turn above the other and has overall dimensions of 1.00 meter I.D. by 1.38 meter O.D. by 0.117 meter height. The turn to turn pitch is $3.68 \mathrm{~cm}$. Sandwiching the test coil above and beneath are two halves of 
the split pair background field magnet. Powered by a motorgenerator supplying a maximum operating current of $1200 \mathrm{~A}$, the magnet is designed to generate a 4 tesla field at the center turn of the test conductor. The total inductance of the background magnet is 21 Henries and at full current the stored energy of the background magnet is substantial, $15 \mathrm{MJ}$. The magnet is equipped with a protection system complete with quench detection circuitry, DC current interrupting switches, and an energy-dump resistor. The test coil, including the $50 \mathrm{kA}$ conductor and the structural backing, the background magnet, and the magnet protection system are all fabricated by Westinghouse.

Instrumentation is distributed throughout the three-turn test coil and provides for a variety of experiments. For the stability tests, an array of nichrome wires, imbedded in the high purity aluminum stabilizer over a $22.2 \mathrm{~cm}$ length, provide the necessary thermal disturbances. Voltage taps and calibrated carbon thermometers, also installed in the aluminum stabilizer, record the voltages and temperatures of the conductor. Additional instrumentation includes bath thermometers, Hall probes, and helium level indicators. Voltage taps over the threeturn test coil and the test-coil/down-lead joints also serve the purpose of detection for safety circuits. The safety circuits automatically turn off the $100 \mathrm{kA}$ power supply if/when any of the detected voltages exceeds a preset value.

Figure 1 shows the assembly of the test coil and background magnet inside the POPE dewar. The dewar itself is made up of two primary parts; an annular inner dewar surrounded by a larger, cylindrical vacuum vessel. The assembled test coil and background coil rest on the bottom of the inner dewar and may be operated at $1.8 \mathrm{~K}$ and 1 atmosphere through the use of a heat exchanger mounted on a $12.7 \mathrm{~cm}$ thick $\mathrm{G}-10$ isolator flange. The fundamental ideas governing the operation of the heat exchanger are the same as those described by Canavan et al. ${ }^{5}$ Above the flange, the reservoir of saturated $4.2 \mathrm{~K}$ helium is maintained at a constant level through the use of a liquid helium keep-full system. All penetrations to the inner dewar are through stand pipes which extend between the cover (and sides) of the inner dewar, and the domed cover of the vacuum vessel. For example, shown in Fig. 1 are the penetrations for the J-T valve stem, the heat exchanger vacuum line, and one of the $75 \mathrm{kA}$ helium vapor-cooled current leads.

Figure 2 shows the arrangement of the POPE dewar and all the other various support systems within the High Current Laboratory at the University of Wisconsin-Madison. Among the noteworthy features are the following: Continuous DC currents, up to $100 \mathrm{kA}$, can be supplied to the test coil from the $100 \mathrm{kA}$ power supply system. The system includes a $4160 \mathrm{~V}$ AC disconnect switch, an $1100 \mathrm{KVA}$ variable voltage transformer, a DC power supply with $100 \mathrm{kA}, 6 \mathrm{~V}$ output, and a $37 \mu \Omega$ watercooled copper bus from the power supply to the dewar. AC ripple on the $\mathrm{DC}$ signal is minimized by the 24 pulse arrangement of the DC power supply, and by using the variable voltage transformer to keep the SCR phase-back angle to less than $10^{\circ}$. A continuous supply of liquid helium to the POPE

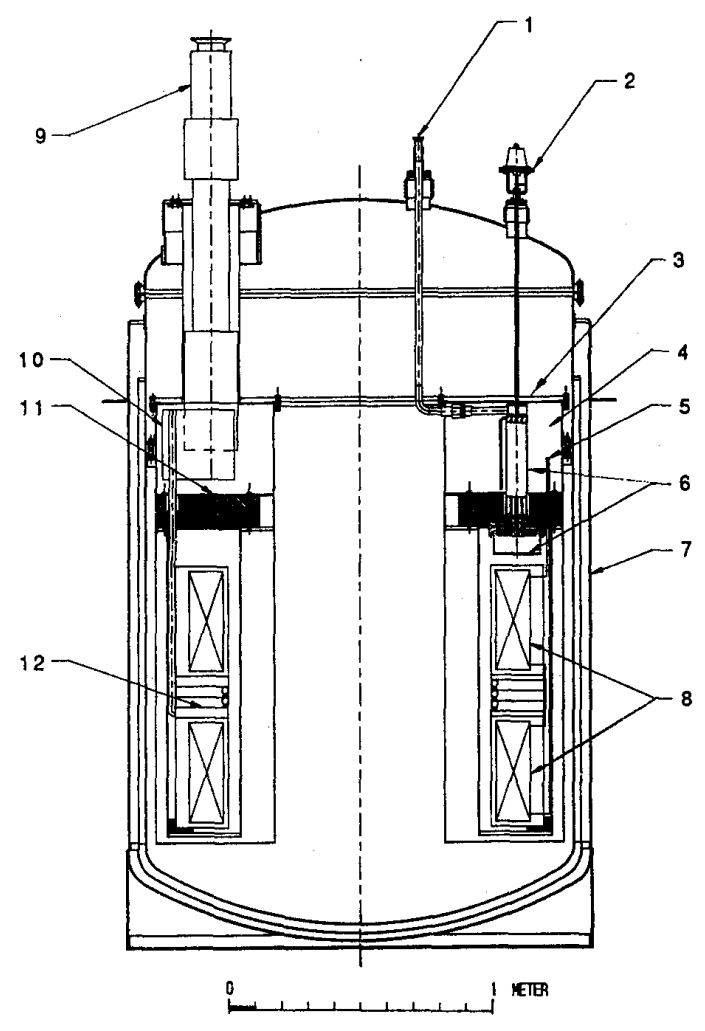

Figure 1. Cross-section of the assembled Proof of Principle Experiment. Numbered details are as follows: 1) Vacuum line for heat exchanger; 2) Joule Thomson valve control; 3) Inner dewar cover; 4) $4.2 \mathrm{~K}$ reservoir; 5) to magnet current leads; 6) Heat exchanger; 7) Vacuum vessel (with radiation shields); 8) Background coil; 9) $75 \mathrm{kA}$ vapor-cooled current lead; 10) $4.2 \mathrm{~K}$ current bus; 11) G-10 isolator flange, 12) Test coil.

dewar is available from an 11,000 gallon dewar parked outside the building. Boil-off from the experiment is either recovered in a gas-bag and compressed for future re-liquification, or it is vented outside the building. The vacuum system provides 1500 cfm of pumping capacity for the heat exchanger. This translates to roughly 25 watts of cooling power for the lower portion of the inner dewar. Twenty-four channels of data are gathered during a run; each at a $1 \mathrm{kHz}$ sampling rate. All the data is fed through floating differential amplifiers, into a MacIntosh II computer equipped with a MacAdios data acquisition system. The details of the data acquisition system are given elsewhere. ${ }^{6}$

\section{Procedure and Results}

\section{Cool Down}

Following various pre-tests of the major components of the POPE, all systems are assembled and the experiment is precooled to $77 \mathrm{~K}$. At this point the experiment is checked for leaktightness and instrumentation integrity, then cooled to $4.2 \mathrm{~K}$. 


\section{Test Coil Current Cycles}

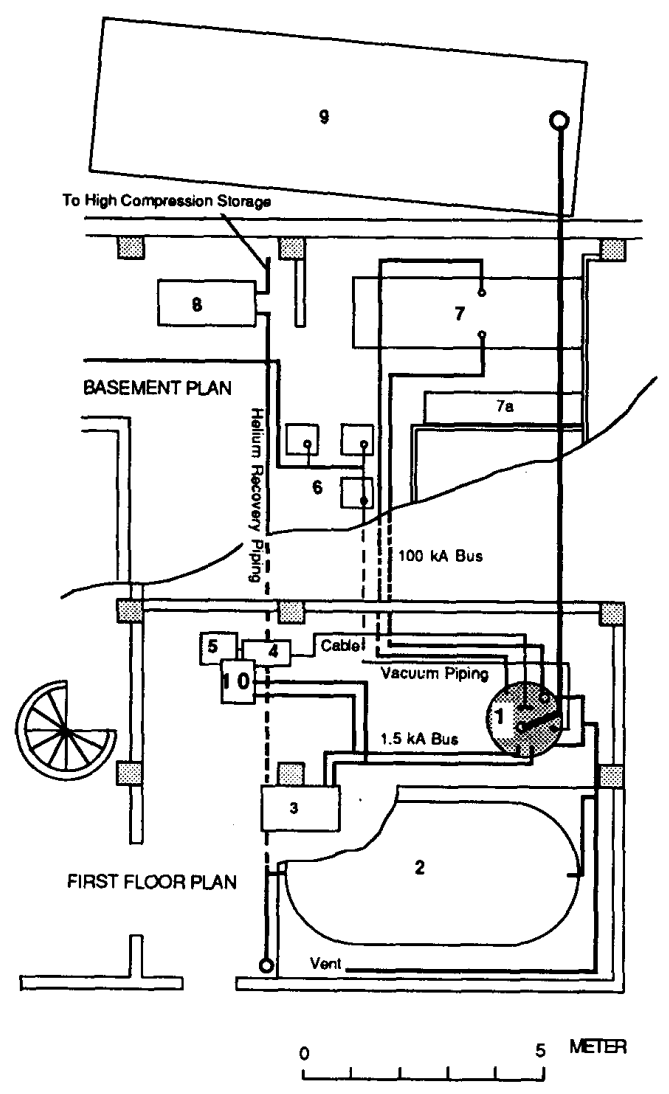

Figure 2. Laboratory facility and support systems built for the POPE. Numbered details are as follows: 1) POPE dewar; 2) Helium recovery gas bag; 3 ) Motor generator power supply; 4) Instrumentation rack; 5) Computer cabinet; 6) Vacuum pumps; 7) $100 \mathrm{kA} \mathrm{DC}$ power supply; 7a) Power supply control unit; 8) Helium recovery compressor; 9) 11,000 gallon helium trailer; 10) Protection system dump resistor.

During the cool-down phase we find that the test conductor's RRR is 1820 .

After filling the dewar with liquid helium, the procedure is to use the heat exchanger to further cool the magnet-test coil region to $1.8 \mathrm{~K}$. This procedure was followed in spite of a small helium leak into the vacuum vessel which developed during the cool down to $4.2 \mathrm{~K}$. As a result of the leak, the vacuum, and consequently the dewar's thermal insulation, slowly degraded over a span of a few days. After reaching a lowest temperature of $2.05 \mathrm{~K}$, the magnet-test coil region gradually began warming. Making the best use of the cold temperature was accomplished by gathering data first in the self field of the test coil alone, and subsequently taking the time to ramp up the background magnet for further data.
Over the course of the few days of testing (and consequently through the temperature range of $2.05 \mathrm{~K}$ to $4.2 \mathrm{~K}$ ) the current in the test coil was cycled to either $50 \mathrm{kA}$ or $60 \mathrm{kA}$ a total of 24 times. The current was held at $50 \mathrm{kA}$ for as long as $11 / 2$ hours; at $60 \mathrm{kA}$ arbitrarily up to 5 minutes. The self field of the test coil is approximately 1.25 tesla in the range of $50 \mathrm{kA}$ to $60 \mathrm{kA}$.

\section{$\underline{\text { Stability Tests }}$}

The procedure carried out for the stability tests is standard: A thermal disturbance is applied to the test conductor by one of the conductor heaters while information from the voltage taps, conductor thermometers, and bath thermometers are recorded prior to, during, and following the thermal disturbance. The pulse energy is applied from a pulsed capacitor bank in the form of a $1 / 2$ sine wave pulse of approximately $5 \mathrm{msec}$ duration. Measurements of the pulsed current and the resultant voltage across the heater are used to determine the delivered energy. A schematic description of the instrumentation used for the stability tests is shown in Fig. 3.

According to the dynamic stability model ${ }^{3,4}$, a traveling normal zone can exist only above some threshold current $I_{t h}$. However, above $I_{t h}$ the traveling normal zone can be initiated by a thermal disturbance just large enough to drive some portion of the conductor above the current sharing temperature $T_{c s}$. At 40 $\mathrm{kA}$ we determine an appropriate disturbance to create a normal zone and observe that the normal zone recovers. Similarly, recovery is observed at $50 \mathrm{kA}$ and $55 \mathrm{kA}$. The identical disturbance energy applied at $60 \mathrm{kA}$ produces a normal zone which does not recover.

Data from the same heat pulse, applied at $56 \mathrm{kA}$ shown in Fig. 4 displays a normal zone first appearing in the heated region (VT-8/9) which takes approximately 1 second to recover. In addition, the normal zone appears at successively farther locations away from the heated zone and recovers quickly. Indeed a voltage at location 13/14 appears 116 milliseconds after the normal zone is initiated at the heater

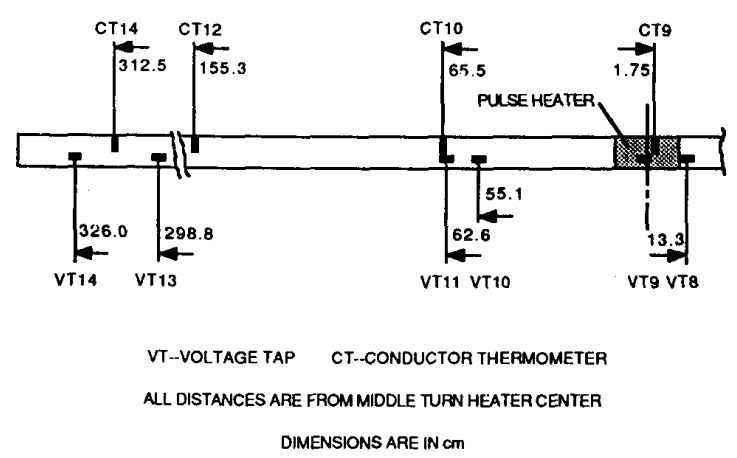

Figure 3. A schematic showing the relative locations of the instrumentation used for the stability tests. 
center, by which time the voltage at location $10 / 11$ recovers to $0.1 \mathrm{mV}$ from a peak value of $0.76 \mathrm{mV}$. In other words, a normal zone of finite length travels away from the disturbed region. This is the traveling normal zone. In fact $\sim 4$ seconds after the disturbance pulse, the normal zone travels through the joint of the test-coil on the down-lead thereby tripping the safety circuit and turning off the $100 \mathrm{kA}$ power supply. A summary of the self field dynamic stability test is shown in Fig. 5 combined with the theoretical calculations ${ }^{4}$ for the conditions of 1.25 tesla (self field) and $2.5 \mathrm{~K}$ bath temperature at which the data was taken. The agreement is very good, in fact, the conductor performs better than calculated; i.e., the measured threshold threshold current is $10 \%$ higher than calculated.

An identical dynamic stability test, conducted with a background field of $1 / 2$ tesla, and at a bath temperature of $3.4 \mathrm{~K}$ reveals a threshold current between $52.5 \mathrm{kA}$ and $55 \mathrm{kA}$. The traveling normal zone is observed and propagates at $25 \mathrm{~m} / \mathrm{s}$ at 55 $\mathrm{kA}$. With these conditions the calculated values are $I_{\text {th }}=47.2$ $\mathrm{kA}$ with a propagation velocity of $21 \mathrm{~m} / \mathrm{s}$ at that current.

\section{Transient Stability}

The transient stability of the test conductor was measured at $50 \mathrm{kA}$, in its own self field, and at a temperature of $3.2 \mathrm{~K}$.

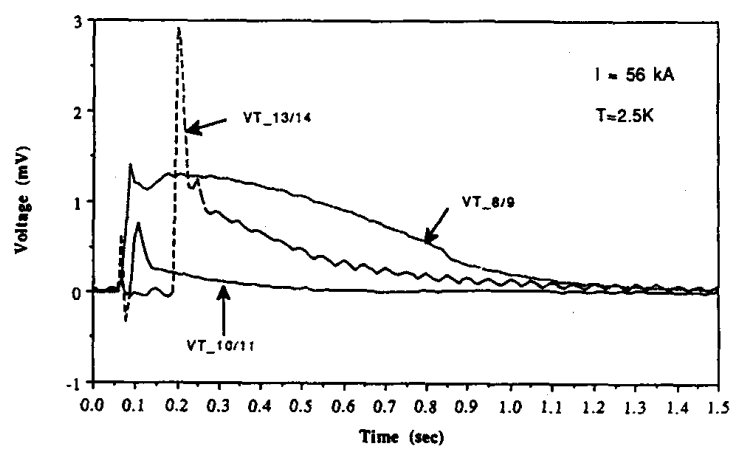

Figure 4. Voltage tap data showing traveling normal zone.

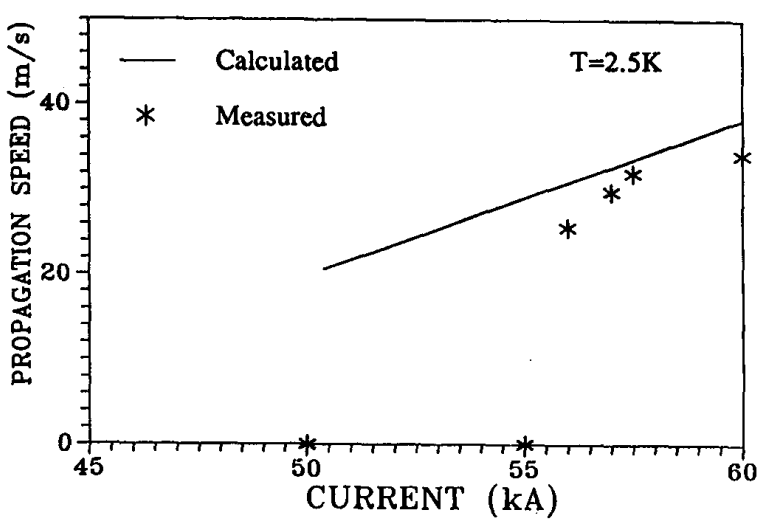

Figure 5. Calculated and measured propagation velocities of traveling normal zones.
Successively larger pulses of energy applied to the conductor all resulted in normal zone recovery. With the largest available pulse from our power supply, the conductor reaches a temperature of $60 \mathrm{~K}$ and nevertheless recovers after 2.7 seconds.

\section{Conclusion}

The test results from the Proof of Principle Experiment confirm the predictions of the dynamic stability model and characterize the conductor's transient stability against a quench. At $50 \mathrm{kA}$ and with a bath temperature of $3.2 \mathrm{~K}$, the conductor is transiently stable even after reaching a temperature of $60 \mathrm{~K}$. A dynamic stability threshold current of $55.5 \mathrm{kA}$ is measured at 2.5 $\mathrm{K}$ and in a self field of 1.25 tesla. Above the threshold current, a traveling normal zone is clearly observed which propagates at velocities in excess of $25 \mathrm{~m} / \mathrm{sec}$. At $3.4 \mathrm{~K}$ and 1.75 tesla, the threshold current is between $52.5 \mathrm{kA}$ and $55 \mathrm{kA}$ and a traveling normal zone propagation velocity of $25 \mathrm{~m} / \mathrm{s}$ is measured at 55 kA. The threshold current measurements are $\sim 10 \%$ higher than calculated values. Both threshold current values and propagation velocities are in accordance with the predicted dependence on temperature and magnetic field. As detailed elsewhere ${ }^{4}$, the same calculations predict a threshold current of $55.5 \mathrm{kA}$ at $1.8 \mathrm{~K}$ and 4.6 tesla, as compared to the ETM expreme conditions of 50 $\mathrm{kA}, 1.8 \mathrm{~K}$ and 4.6 tesla. Thus the conductor is shown to be dependable for ETM operating conditions for which normal zone propagation could not occur.

\section{Acknowledgements}

This work was supported by DNA contract DNA001-88C-0027 and EPRI contract RP2988-1.

\section{$\underline{\text { References }}$}

1. O. Christianson, "Normal Zone Evolution and Propagation in a Cryogenically Stable Superconductor," Adv. in Cryo. Eng., Vol. 31, pp. 383-390, 1986.

2. X. Huang, Y. M. Eyssa and M. A. Hilal, "Stability Analysis of Round Conductors Cooled by $1.8 \mathrm{~K} \mathrm{He} \mathrm{II,"} \mathrm{Internal}$ memo to UW-ASC group, July $23,1988$.

3. L. Dresner, "Propagation of Normal Zones of Finite Size in Large Composite Superconductors," Paper LB-03 presented at the 11th Int. Conference on Magnet Technology, August 1989.

4. X. Huang and Y. M. Eyssa, "Stability of Large Composite Superconductors," Paper LMP-24 presented at this conference 1990 Applied Superconductivity Conference.

5. E. R. Canavan, D. C. Larbalestier and S. W. Van Sciver, "A 13T-1.8 K NbTi Laboratory Test Coil," IEEE Trans. on Magnetics, 24 (no. 2), March 1988, pp. 1082-1085.

6. J. M. Pfotenhauer, "Superconductor Stability using MacAdios II and Lightspeed C," GW Instruments Application note \#18, Somerville, MA, February 1990. 